\title{
Actualización en clínica y terapia de mordedura de serpiente (ofidismo)
}

\author{
Update on snake bites
}

\author{
Ciro Maguiña-Vargas ${ }^{1,2, a}$, Omayra Chincha-Lino ${ }^{2, b}$, Pierina Vilcapoma-Balbín ${ }^{2, b}$, Diana Morante ${ }^{2, b}$
}

\section{RESUMEN}

Esta revisión sobre mordedura de serpiente (ofidismo), buscar actualizar la epidemiologia clínica y terapia de la patología frecuente en la selva y costa peruana. Entre las serpientes venenosas destaca el accidente producido por el género Bothrops, en el Perú la especie B. atrox (Jergón de la selva) es el causante en más del 90\% de los casos.

PALABRAS CLAVE: Mordedura de serpiente, Bothrops, epidemiología. (Fuente: DeCS BIREME).

\section{SUMMARY}

This review on snake bites, seek to update the clinical epidemiology and therapy of frequent pathology in the Peruvian jungle and coast. Among the venomous snakes, the accident caused by the genus Bothrops stands out, in Peru the species B. atrox (jungle jar) is the cause in more than $90 \%$ of cases.

KEYWORDS: Snake bites, Bothrops, epidemiology. (Source: MeSH NLM).

\section{INTRODUCCIÓN}

La mordedura de serpiente (ofidismo), es el síndrome causado por la inoculación de sustancias venenosas de alguna de las serpientes venenosas (1). Existen unas tres mil especies de serpientes en el mundo, de ellas al menos el $10 \%$ son venenosas para el ser humano. Las familias de serpientes consideradas venenosas son cinco: Viperidae, Elapidae, Hydrophidae, Atractaspidae y Colubridae (2).

En el Perú, los nombres de las serpientes están en relación con las aéreas geográficas y cada una adopta un nombre diferente, así tenemos: el género Lachesis (shushupe) se encuentra solo en la selva, el género Crotalus (cascabel) se encuentra en Sandia (Puno) y Tambopata (Madre de Dios), los géneros Bothrops (jergón) y Micrurus (corales) se encuentra en la costa, sierra y selva (3). Desde el año 1966 se vienen realizando estudios de la fauna silvestre ponzoñosa peruana, habiéndose identificado hasta la fecha de edición del presente documento, 38 especies de serpientes venenosas, del complejo Grupo Bothrops (19 especies), Crotalus (1 especie), Lachesis (1 especie), Micrurus (16 especies) y Pelamis (1 especie), de estas especies identificadas al menos son 7 son oriundas del Perú y las demás son comunes a otros países vecinos (4).

\section{Epidemiología}

La Organización Mundial de la Salud (OMS) estima que ocurren 2500000 accidentes ofídicos por año, resultando en 125000 muertes a nivel mundial y 100000 sobrevivientes con secuelas severas; América Latina después de África, es el área más afectada (5).

\footnotetext{
Facultad de Medicina Alberto Hurtado, Universidad Peruana Cayetano Heredia. Lima, Perú.

Departamento de Enfermedades Infecciosas Tropicales y Dermatológicas, Hospital Cayetano Heredia. Lima, Perú.

Médico Infectólogo, Tropicalista, Dermatólogo;

Médico Especialista en Enfermedades Infecciosas Tropicales.
} 
En el Perú, en el año 2017 se registraron 2103 casos con una incidencia por 10000 habitantes de 0,68 y solo se reportó una muerte por la misma causa; la mayoría de casos $(66 \%)$ se siguen registrando en ciudades de la selva: Loreto, San Martin, Ucayali y Junín $(4,6)$. Estudios epidemiológicos actuales concuerdan en que los accidentes ofídicos afectan con mayor frecuencia a las personas entre los 18 a 59 años $(60 \%)(4,7)$. El género masculino es el afectado con mayor frecuencia, debido a las actividades agrícolas, de recolección y caza que realizan $(4,7)$. Los accidentes ocurren con mayor frecuencia entre las 9-12 horas y 17 - 20 horas (costumbres nocturnas de la especie vipéridas) (1). Más de la mitad de los accidentes ofídicos se producen entre noviembre a marzo (meses lluviosos) de la selvas alta y baja $(1,7,8)$.

En Perú, las serpientes del género Bothrops son las causantes más frecuentes de ofidismo, siendo las especies más comunes de este género son $B$. atrox (jergón) y $B$. bilineatus (loro machaco), en las zonas selváticas, $B$. barnetti (macanche), en la costa norte y $B$. pictus (víbora de costa), en las costas centro y sur $(1,3,4,7,8)$. Los casos de ofidismo por serpientes del género Lachesis son poco frecuentes; en la selva peruana habita la Lachesis muta muta (shushupe) $(1,8)$. No hay casos reportados de accidentes causados por serpientes de la familia Elapidae (corales) e Hydrophiidae (serpientes marinas), hasta el momento del presente reporte $(4,9)$.

En el Hospital Cayetano Heredia se han tenido entre los años 2009 y 2018, 42 pacientes hospitalizados por cuadro de ofidismo, la edad media fue 31,16 años (rango: $1-79$ años) y el $71,43 \%$ fueron de sexo masculino. De 20 historias revisadas, recibieron suero antiofídico: 20/20 (100\%), reportándose complicaciones en 7/20 (35\%), destacando el síndrome compartimental en 4 casos, trastorno de la coagulación en dos y síndrome neurotóxico en un caso y la estancia hospitalaria promedio fue 6,45 días. No se registraron casos mortales (comunicación personal Dra. Morante).

\section{Características del veneno}

El veneno es una secreción exocrina de las glándulas salivales y cumple funciones digestiva y defensiva. El veneno, por lo general, es usado para inmovilizar las presas, matarlas y comenzar la digestión, el veneno está constituido por varios péptidos y proteínas tóxicas, como miotoxinas, hemorraginas, neurotoxinas y toxinas coagulantes (4).
El veneno es viscoso, debido al contenido de sólidos totales (aproximadamente 25\%). De estos sólidos, el $70-90 \%$ corresponde a proteínas y polipéptidos de peso molecular elevado; estas proteínas son las que ocasionan la mayoría de los efectos biológicos. El 10 a $30 \%$ de los solutos restantes corresponde a una amplia gama de sustancias orgánicas de peso molecular bajo (carbohidratos, péptidos pequeños, aminoácidos libres, aminas biógenas, nucleótidos), compuestos inorgánicos y elementos aniónicos y catiónicos. De acuerdo al género de la serpiente, el veneno puede tener diferentes acciones fisiopatológicas (tabla 1) $(1,4)$. La complejidad de las fracciones tóxicas ocasiona que los pacientes puedan desarrollar diversos efectos, que dependen de muchos factores como, por ejemplo, la cantidad de veneno inoculado, la zona del cuerpo, el tamaño de herida, el tamaño de la serpiente y la edad del paciente. Las características del veneno también son importantes, ya que la absorción de las toxinas se realiza por vía linfática, y los venenos neurotóxicos y hemolíticos se absorben más rápido que los proteolíticos y hemorrágicos $(1,4)$.

\section{Manifestaciones clínicas}

La gravedad del cuadro clínico está en relación a la susceptibilidad del paciente al veneno, así como a la cantidad inoculada (1). La zona afectada depende de la serpiente. Las serpientes arborícolas producen mordeduras con más frecuencia en los miembros superiores y la cabeza y las terrestres, en los miembros inferiores (10). Además, los accidentes en la selva ocurren más en los miembros inferiores y en la costa en los miembros superiores. Esta diferencia se ha atribuido a la actividad realizada por el paciente durante la mordedura (7).

Tabla 1. Acciones de los venenos según género (1).

\begin{tabular}{ll}
\hline GÉNERO & \multicolumn{1}{c}{ ACCIÓN } \\
\hline \multirow{3}{*}{ Bothrops, Bothriopsis, } & Proteolítica \\
Porthidium & Coagulante \\
& Nefrotóxica \\
& Vasculotóxica \\
& Proteolítica \\
Lachesis & Coagulante \\
& Vasculotóxica \\
& Vagal \\
& Miotóxica Coagulante \\
& Neurotóxica \\
Crotalus & Nefrotóxica \\
& Vasculotóxica \\
Micrurus & Neurotóxica \\
\hline
\end{tabular}


En general, se recomienda evaluar al paciente luego de cuatro a seis horas, para determinar la gravedad y estadio clínico (sobre todo en caso de accidentes por Bothrops). Cabe señalar que esto no impide la administración temprana de la sueroterapia específica. El envenenamiento, por tratarse de proteínas extrañas altamente antigénicas, puede provocar reacciones anafilácticas, sobretodo en pacientes con mordeduras previas, por lo cual debe tenerse al lado medicación para un shock anafilactico de presentarse el evento (1).

De acuerdo a la serpiente, los cuadros clínicos de los accidentes ofídicos se clasifican en botrópicos, crotálicos, lachésicos, elapídicos y por serpiente marina. Cada tipo de accidente ofídico tiene su escala de gravedad (tabla 2).

\section{Accidente botrópico}

Accidente ofídico producido por las serpientes de los géneros Bothrops, Bothriopsis, Bothriechis y
Bothrophias. En el Perú, más del 95\% de los accidentes son causados por serpientes de estos géneros. El cuadro clínico después de una a tres horas de la mordedura se caracteriza por dolor local intenso, edema firme, que aumenta progresivamente, y eritema con manchas rosáceas o cianóticas. Además, si la cantidad de veneno inoculado es mayor, en las primeras horas el paciente presentará disminución de la presión arterial y del fibrinógeno circulante, lo que causa equimosis, linfangitis, bulas y, luego de días o semanas, necrosis superficial o profunda del miembro o zona afectada, que puede causar una necrosis total. El paciente puede presentar epistaxis, gingivorragia, hematuria, melena, hematemesis, hemoptisis y sangrado de otros órganos. La gravedad de la lesión dependerá de la progresión de la misma, si es que se queda solo en un miembro (mano, pie) y sin trastornos en la coagulación y es leve (figura 1A), o si progresa a más de un miembro con alteración de las pruebas de coagulación y es moderada (figura 1B); y si el paciente presenta choque, falla multiorgánica y sangrado espontáneo en caso severo (figura 2) $(4,9,11)$.

Tabla 2. Clasificación de la severidad según tipo de accidente ofídico (11).

\begin{tabular}{|c|c|}
\hline Tipo de Accidente & Severidad del Envenenamiento \\
\hline \multirow{3}{*}{ Botrópico } & $\begin{array}{l}\text { Leve: Si después de } 6 \text { horas de observación la reacción local es leve con edema localizado } \\
\text { en la zona agredida y no se presentan síntomas de compromiso sistémico, ni hay variaciones } \\
\text { importantes en las pruebas de laboratorio. }\end{array}$ \\
\hline & $\begin{array}{l}\text { Moderado: Cuando el tiempo de coagulación se presenta prolongado o incoagulable, leucocitosis } \\
\text { con neutrofilia moderada con presencia de edema que tiende a extenderse, dolor, equimosis. Puede } \\
\text { haber gingivorragia, proteinuria y hematuria moderada. }\end{array}$ \\
\hline & $\begin{array}{l}\text { Severo: Si la sangre es incoagulable a pesar del tratamiento específico inicial. Reacción local } \\
\text { intensa con edema progresivo, equimosis, flictenas y equimosis a distancia. Se presenta hematuria } \\
\text { con oliguria y anuria, gingivorragia, epistaxis y melena. Hay riesgo de shock por colapso periférico } \\
\text { dentro de las primeras } 24 \text { horas; además la disminución súbita del hematocrito pone en evidencia } \\
\text { hemorragia interna. El riesgo de necrosis es alto. }\end{array}$ \\
\hline \multirow{3}{*}{ Crotálico } & Leve: Si después de las 3 horas no hay facies neurotóxica. \\
\hline & $\begin{array}{l}\text { Moderado: Si hay facies neurotóxica y mioglobinuria moderada sin daño renal, ni parálisis } \\
\text { respiratoria. }\end{array}$ \\
\hline & $\begin{array}{l}\text { Severo: Si se presenta facies neurotóxica que aparece en las primeras } 6 \text { horas, si hay mioglobinuria } \\
\text { y anuria severa, la muerte puede ocurrir dentro de las primeras } 24 \text { horas. En los casos tratados con } \\
\text { suero antiofídico en dosis suficiente dentro de las primeras horas el riesgo de muerte disminuye. }\end{array}$ \\
\hline \multirow{3}{*}{ Lachésico } & $\begin{array}{l}\text { Leve: Si después de } 6 \text { horas la reacción local cutánea es leve y no hay alteraciones en los exámenes } \\
\text { de laboratorio. }\end{array}$ \\
\hline & $\begin{array}{l}\text { Moderado: Cuando hay equimosis, edema, dolor progresivo, hay alteración de la presión arterial } \\
\text { (hipotensión) y hay alteración de la conciencia (agitación, somnolencia). }\end{array}$ \\
\hline & $\begin{array}{l}\text { Severo: Cuando el edema, dolor, equimosis y flictenas aparecen en forma progresiva, hay } \\
\text { hipotensión arterial, nauseas, vómitos, diarrea y la sangre incoagulable. }\end{array}$ \\
\hline \multirow{3}{*}{ Elapídico } & Leve: $\mathrm{Si}$ antes de los 30-60 minutos no presenta facies neurotóxica. \\
\hline & Moderado: Si en las dos primeras horas se presenta la facies neurotóxica, sin dificultad respiratoria. \\
\hline & $\begin{array}{l}\text { Severa: Si se presenta facies neurotóxica con dificultad para respirar y deglutir, y estado de coma. } \\
\text { La muerte puede sobrevenir en las } 4-6 \text { horas posteriores a la mordedura. }\end{array}$ \\
\hline
\end{tabular}




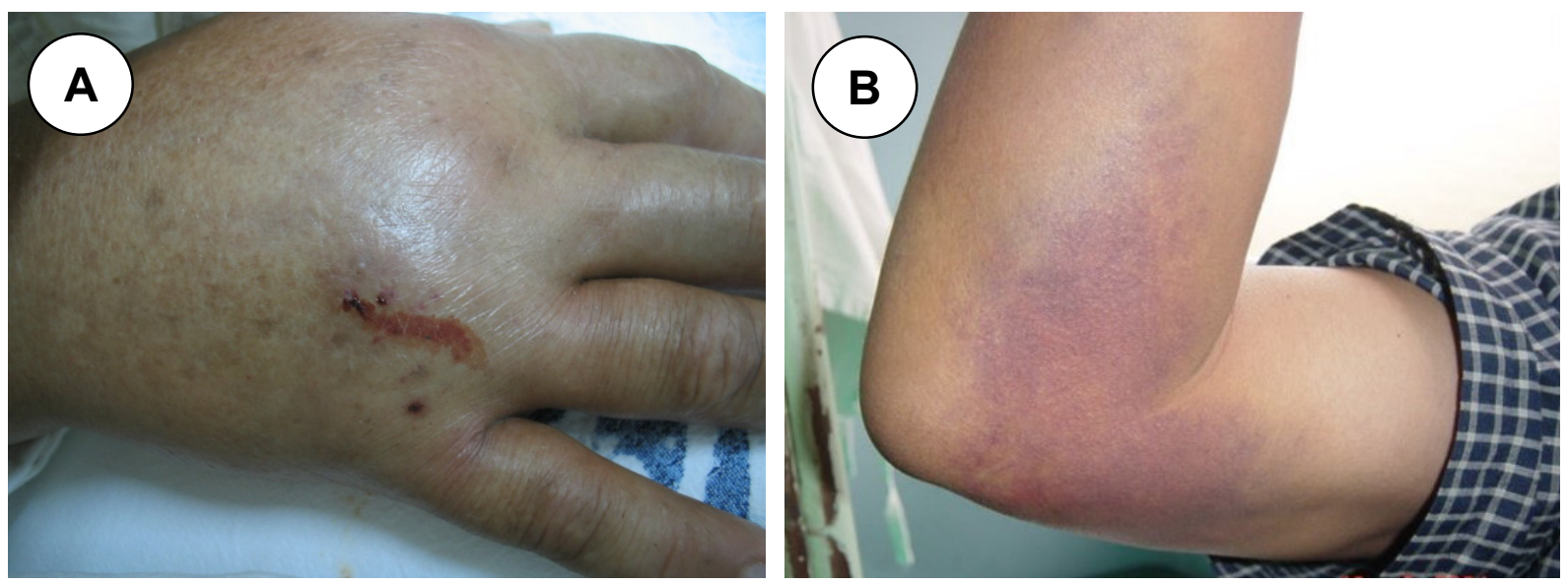

Figura 1. Accidente ofídico por Bothrops. A: Leve; B: Con severa equimosis.

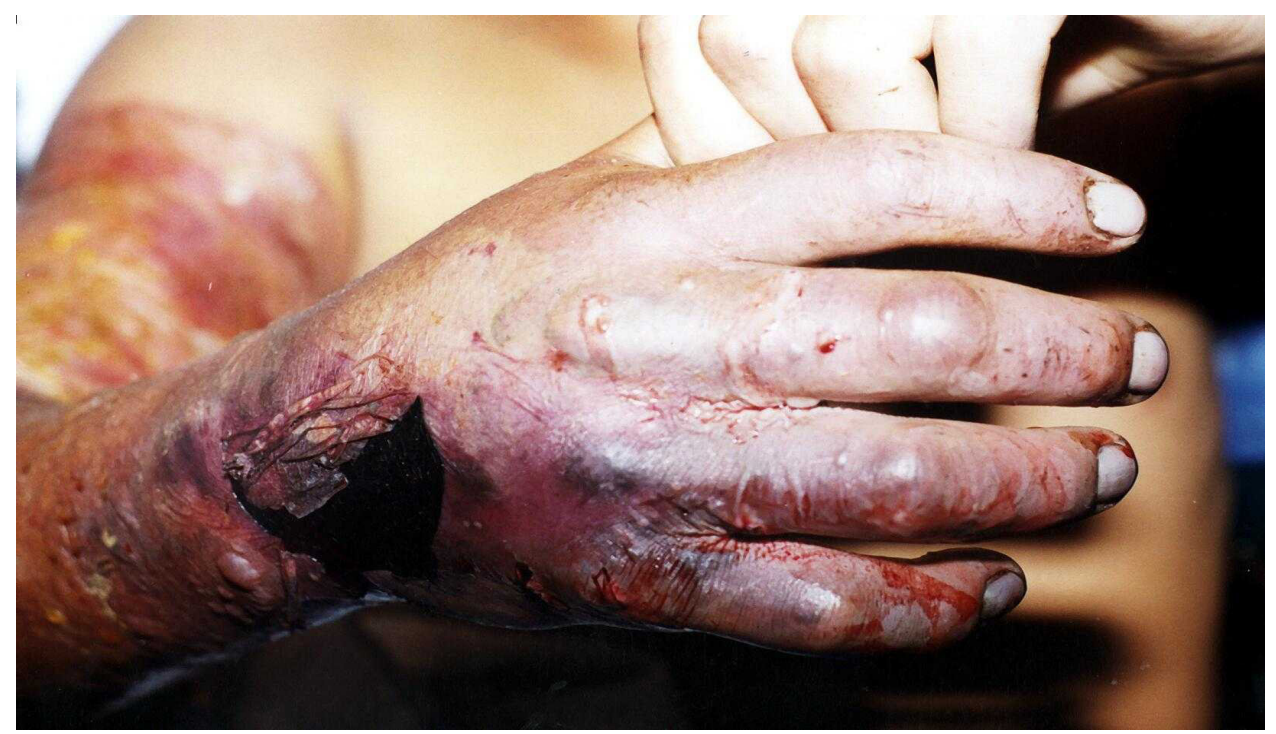

Figura 2. Accidente botrópico de la selva, complicado con gangrena.

\section{Accidente crotálico}

Producido por una serpiente de la especie Crotalus durissus ssp. El cuadro clínico se caracteriza por dolor local leve que desaparece rápidamente. Además, se presenta parestesia, mialgia generalizada, disnea progresiva, taquicardia, diplopía y obnubilación. Luego de 30-60 minutos, el paciente presenta facies neurotóxica, caracterizada por caída de los párpados y trastornos de la visión (visión doble y confusa, que puede llegar hasta la ceguera temporal). La orina se oscurece gradualmente (mioglobinuria) y además disminuye en volumen (oliguria). Puede llegar a anuria y falla renal aguda. El accidente crotalico tiene mayor letalidad a pesar que el accidente por esta serpiente no es frecuente $(4,9,10)$.

\section{Accidente lachésico}

Este accidente es causado por la serpiente Lachesis muta muta, se caracteriza por confusión mental (acción vagal), palidez intensa, sudoración, frialdad cutánea y obnubilación que se observa luego de las primeras horas del accidente, posteriormente se desarrolla hipotensión, que puede ocasionar shock e incluso muerte. Si el paciente tolera las primeras horas, luego de 8-12 horas la presión arterial se normaliza. La letalidad no es mayor que en el accidente botrópico $(4,9)$.

\section{Accidente elapídico}

Ofidismo causado por las serpientes del género Micrurus. No produce una lesión cutánea importante, 
solo adormecimiento en la región lesionada. A los 30 a 60 minutos del accidente, aparece la facies neurotóxica, sialorrea, disfagia y, a veces, disartria. También aparecen parálisis flácida del sistema locomotor y alteraciones en la función miocárdica. De manera más tardía, se presentan alteraciones urinarias y hematuria, que pueden progresar a oliguria, anuria e insuficiencia renal aguda $(4,9,12)$.

\section{Accidente por serpiente marina}

Este accidente es producido por las serpientes de la familia Hidrophidae. Estas serpientes son poco agresivas y tienen solo una pequeña cantidad de veneno. Al momento de la mordedura, no hay dolor local, pero después de una hora hay dolores musculares generalizados al realizar movimientos; luego, aparecen parálisis muscular, sudor y vómitos; 30 minutos después empieza un cuadro hemolítico y síntomas neurotóxicos. La presión arterial puede estar normal o elevada. La muerte se produce entre las dos y tres horas, por parálisis respiratoria precedida de obnubilación y coma, con pupilas dilatadas y desaparición de reflejo a la luz. No se ha reportado ningún caso de este tipo de accidentes ofídicos en los últimos años.

\section{Diagnóstico y diferencial}

El antecedente de haber sido atacado por una serpiente está casi siempre presente. En caso no se haya podido ver al animal agresor, se deberá descartar a otros animales como agresores $(1,4,9)$. Si el paciente refiere haber sufrido una mordedura por serpiente, el diagnóstico diferencial incluye la diferenciación de la serpiente agresora. Existen diversas características de las serpientes que ayudan a determinar la especie. Además, se debe tener en cuenta la fauna ofídica del área geográfica donde se produjo el accidente $(1,4,9)$. Debe tenerse en cuenta, la posibilidad de un seudoofidismo, accidente en el que la serpiente no es venenosa o en el que no se ha producido inoculación de veneno (13).

\section{Complicaciones}

Existen diversas complicaciones, entre las médicas, están la insuficiencia renal aguda, causada por especies del género Crotalus $\mathrm{y}$, en menor frecuencia, por especies del género Bothrops, La insuficiencia respiratoria aguda, en accidentes elapídicos y crotálicos; el choque, producido en accidentes botrópico, lachésico y eventualmente en los crotálicos; las infecciones locales (flora polimicrobiana), principalmente abscesos, causadas en accidentes botrópico y lachésico.

Las complicaciones quirúrgicas son el síndrome compartimental y la gangrena. En la primera, se debe realizar una fasciotomía y, si existe un trastorno de la coagulación, hacer transfusiones sanguíneas. La evidencia de gangrena hace necesaria la amputación.

\section{Tratamiento}

\section{Atención extrahospitalaria}

En el caso de un accidente ofídico se debe tener en cuenta las consideraciones siguientes:

1. Tratar el caso como una emergencia, tranquilizar e inmovilizar al paciente, lavar la zona de la mordedura con agua y jabón, inmovilizar la parte afectada, mediante el empleo de férulas, entablillados u otros, trasladar al paciente al centro o puesto de salud más cercano (cargado o en camilla), manteniendo el miembro afectado a un nivel más alto que el eje del cuerpo, hidratar al paciente, no usar bebidas con alcohol ni medicamentos, no aplicar torniquetes ni ligaduras en el miembro afectado, no hacer cortes ni succionar el veneno, ni utilizar medidas caseras, como el uso de hielo, corriente eléctrica, kerosene $\mathrm{u}$ otros, colocar el miembro afectado en una posición elevada con respecto al cuerpo de 30 a 45 grados y con un arco protector. En caso de otras partes del cuerpo mantener al paciente en posición horizontal, medir diariamente el o los miembros afectados, lo que permitirá realizar el diagnóstico oportuno del síndrome compartimental para su derivación inmediata y evaluar la necesidad de la fasciotomia; en el caso de hematomas que no se resuelven, solicitar ecografía y ante el riesgo de síndrome compartimental, realizar la interconsulta al Servicio de Cirugía, considerar el manejo del dolor, preferentemente paracetamol, tramadol, metamizol, no utilizar AINES (ibuprofeno, naproxeno, diclofenaco, entre otros), aplicar vacuna antitetánica después de controlado el sangrado.

2. No administrar heparina, gluconato de calcio, considerar el uso de suero antiofídico específico, independientemente si lo recibió anteriormente, teniendo a la mano los medicamentos necesarios para el tratamiento de una posible reacción de hipersensibilidad (adrenalina), Anotar la hora 
del accidente, el orden de aparición de síntomas y la evolución, los rasgos de la serpiente, la situación geográfica y la actitud del ofidio. Notificar del accidente e iniciar el traslado $(4,9)$.

\section{Atención intrahospitalaria}

Se debe evaluar en primer lugar el grado de envenenamiento y el tiempo de la evolución para considerar aplicación de suero antiofídico de ser necesario y aplicar medidas de apoyo: crioterapia, revisión del estado de inmunidad antitetánica, analíticas sanguíneas que incluyan electrocardiograma, tiempos de coagulación incluyendo tiempo de protrombina, examen de orina que evalúe proteinuria y hematuria y se debe marcar el edema del miembro afectado para la evaluación de la progresión, la cual debe evaluarse cada 60 minutos y en estado intrahospitalario.

\section{Suero antiveneno}

En el caso de un accidente ofídico por Bothrops, considerar el uso de suero antiofídico específico y la posible reacción de hipersensibilidad. El miembro afectado se debe colocar en posición fisiológica y con un arco protector. Puede administrarse analgésicos para el manejo de dolor. Según el caso, se aplicará la vacuna antitetánica o antitoxina (13).

En los accidentes por serpientes de los géneros Micrurus y Crotalus, además de lo recomendado para los accidentes por Bothrops, se debe considerar: respiración artificial, cardiotónicos, anticolinesterásicos (en caso de accidentes por especies del género Micrurus) y diálisis (en la insuficiencia renal, por accidentes por especies del género Crotalus) $(1,4,9)$.

El suero antiofídico neutraliza específicamente el veneno circulante contra el cual fue elaborado, mas no tiene acción sobre las lesiones ya producidas antes de su aplicación. El suero debe ser aplicado rápidamente, dentro de las primeras 24 horas del envenenamiento, de persistir alterado las pruebas de coagulación y deterioro clínico se puede usar el suero más días. El uso del suero tiene indicación absoluta en caso de embarazadas, síndrome compartimental y las dosis en los niños es igual que en los adultos. En caso no haya inoculación de veneno (mordedura seca) ni evidencia clínica o de laboratorio de envenenamiento, se recomienda no administrar el suero antiofídico. Como este suero es un producto biológico heterólogo elaborado a partir de plasma equino, el paciente puede presentar reacciones alérgicas. Se recomienda tener disponibles el equipo y materiales necesarios para prevenir una respuesta alérgica por la administración del suero.

\section{Tratamiento especifico}

En Perú, el Instituto Nacional de Salud produce tres tipos de suero: suero antibotrópico polivalente líquido, suero anticrotálico monovalente líquido y suero antilachésico monovalente líquido. Estos sueros son preparados con venenos nativos y son altamente específicos para el tratamiento de accidentes ofídicos por serpientes que habitan en Perú $(9,11,12)$ (tabla 3).

Tabla 3. Sueros antiofídicos producidos en el Instituto Nacional de Salud del Perú (4).

\begin{tabular}{|c|c|c|c|}
\hline SUERO & PRESENTACIÓN & EFECTO & SERPIENTES \\
\hline $\begin{array}{l}\text { Suero antibotrópico } \\
\text { Polivalente }\end{array}$ & $\begin{array}{l}\text { Solución inyectable en } \\
\text { frascos por } 10 \mathrm{ml}\end{array}$ & $\begin{array}{l}\text { Capaz de neutralizar no } \\
\text { menos de } 25 \mathrm{mg}\end{array}$ & $\begin{array}{l}\text { Bothrops (B. atrox o jergón } \\
\text { de la selva; B. brazili o jergón } \\
\text { shushupe, B. pictus o jergón de la } \\
\text { costa, B. barnetti o macanche) y } \\
\text { Bothrocophias hyoprorus o jergón. }\end{array}$ \\
\hline $\begin{array}{l}\text { Suero antibotrópico } \\
\text { liofilizado }\end{array}$ & $\begin{array}{l}\text { Frasco ampolla con polvo } \\
\text { liofilizado y un frasco } \\
\text { ampolla con agua estéril } \\
\text { para inyectable por } 10 \mathrm{ml}\end{array}$ & $\begin{array}{l}\text { Capaz de neutralizar no } \\
\text { menos de } 25 \mathrm{mg}\end{array}$ & $\begin{array}{l}\text { Bothrops (B. atrox o jergón } \\
\text { de la selva; B. brazili o jergón } \\
\text { shushupe, B. pictus o jergón de la } \\
\text { costa, B. barnetti o macanche) y } \\
\text { Bothrocophias hyoprorus o jergón. }\end{array}$ \\
\hline Suero antilachésico & $\begin{array}{l}\text { Solución inyectable en } \\
\text { frascos por } 10 \mathrm{ml}\end{array}$ & $\begin{array}{l}\text { Capaz de neutralizar no } \\
\text { menos de } 25 \mathrm{mg}\end{array}$ & Lachesis muta \\
\hline Suero anticrotálico & $\begin{array}{l}\text { Solución inyectable en } \\
\text { frascos por } 10 \mathrm{ml}\end{array}$ & $\begin{array}{l}\text { Capaz de neutralizar no } \\
\text { menos de } 15 \mathrm{mg}\end{array}$ & Crotalus durissus \\
\hline
\end{tabular}


Un estudio previo muestra que, para preservar el suero, se debe mantener a una temperatura menor de $25{ }^{\circ} \mathrm{C}(4,12)$. Las serpientes peruanas inyectan en promedio 20 a $30 \mathrm{mg}$ de veneno por mordedura. El suero antiofídico peruano neutraliza en promedio 25 a $40 \mathrm{mg}$ de veneno por frasco, según el fabricante. Se debe aplicar la cantidad necesaria de suero para neutralizar 30 a $100 \mathrm{mg}$ de veneno ofídico. En las especies que inyectan grandes cantidades de veneno (por ejemplo, Lachesis muta muta y Bothrops brasili), las dosis de suero deben ser mayores $(4,9)$.

En los casos de accidentes botrópicos, crotálicos y lachésicos, el uso de suero antiofídico específico administrado en dosis suficiente y dentro de las primeras horas reduce el riesgo de muerte. En caso de accidente botrópico, además, disminuye la tasa de necrosis (9).

El suero antiofídico se aplica por vía intravenosa, lentamente (en treinta a sesenta minutos), diluido en $500 \mathrm{ml}$ de cloruro de sodio al $0,9 \%$ o en dextrosa al $5 \%$. Durante la aplicación del suero se debe observar al paciente (9). En los niños, el volumen diluyente (dextrosa o cloruro de sodio) debe reducirse a 100 $\mathrm{ml}(4,9)$. En los casos de mordedura sin inoculación de veneno (mordedura seca) y de no encontrarse evidencia clínica o de laboratorio de envenenamiento, no emplear suero antiofídico.

Considerando que el suero antiofídico es un producto biológico heterólogo elaborado a partir de plasma equino, se debe tener el equipo y materiales necesarios para manejar reacciones de hipersensibilidad: adrenalina, corticoides (dexametasona), antihistamínicos (clorfeniramina). En cada caso se debe evaluar el cuadro clínico y revisar la literatura proporcionada por el fabricante para aplicar la cantidad necesaria de frascos. En el Perú, donde predomina básicamente el accidente por Botrops, se requiere un minino de 2 frascos/paciente y dependiendo de la severidad del daño entre leve, moderado y grave, se aumentará la dosis. En los accidentes por Lachesis, Crotalus y Elapídicos los cuadros usualmente son severos.

El número de viales a utilizar en los accidentes por serpientes, se muestran en la tabla 4.

El uso de corticoides es controversial (14), se recomienda dexametasona $(0,3$ a $0,5 \mathrm{mg} / \mathrm{kg}$ peso), por dos días. Un estudio encontró relación entre el uso de corticoides por más de cinco días con una mayor frecuencia de celulitis (7).

En las heridas infectadas se ha encontrado diversos gérmenes como los bacilos aerobios Gram negativos (Morganella morganii, Escherichia coli, Proteus retigeri, Klebsiella spp., Enterobacter spp., Enterococcus faecalis, Aeromonas hydrophila, Pseudomonas aeruginosa, Acinetobacter spp), anaerobios estrictos como Clostridium spp., y una menor proporción de cocos Gram positivos: Staphylococcus epidermidis y S. aureus, Streptococcus spp. Es por ello se pueden usar, en casos leves Amoxicilina/Acido clavulanico VO por 7 días o Ciproflcoaxicna mas clindamicina VO por 7 días, en casos moderados Clindamcina más ceftriaxone EV durante la hospitalización, luego VO por 10 a 14 días según evolución (4).

Tabla 4. Dosificación de sueros antiofídicos.

\section{ACCIDENTE BOTROPICO}

Accidente leve

Accidente moderado

Accidente severo

\section{ACCIDENTE LACHESICO}

Todo accidente se considera como severo

\section{ACCIDENTE ELAPIDICO}

Todo accidente se considera como severo

\section{ACCIDENTE CROTALICO}

Todo accidente se considera como severo $\quad 8-12$ viales
$10-12$ viales

$$
\begin{aligned}
& 2-4 \text { viales } \\
& 5-8 \text { viales } \\
& 9-12 \text { viales }
\end{aligned}
$$

$$
8 \text { - } 12 \text { viales }
$$




\section{Pronóstico}

Los factores que condicionan un peor pronóstico son la mayor longitud del animal atacante, la administración más tardía de la sueroterapia específica, el paciente menor de nueve años de edad y el inicio del tratamiento después de ocho horas del accidente.

La mortalidad se ha asociado a pacientes mayores de cincuenta años y un diagnóstico tardío de complicaciones. La amputación del miembro afectado, una de las secuelas más temidas, se encuentra relacionada con alteraciones de la coagulación, localización de lesiones en los dedos, el desarrollo de ampollas y abscesos, la instalación de insuficiencia renal y el inicio del tratamiento después de diez horas del accidente. La mortalidad en el Perú es baja, menos de uno a dos por ciento $(4,7,9)$.

Por otra parte, la aparición de abscesos parece estar en relación con la disminución de la protrombina y de un fibrinógeno menor de $100 \mathrm{mg} / \mathrm{dl}$.

\section{Correspondencia:}

Ciro Maguiña-Vargas

Correo electrónico: ciro.maguina@upch.pe

\section{REFERENCIAS BIBLIOGRÁFICAS}

1. Manrique H. Ofidismo. Lima: Ministerio de Salud; 2000. (Citado el 29 de enero del 2020) Disponible en: http://bvs.minsa.gob.pe/local/ogei/807_ms-oge115. pdf

2. Norris RL, Auerbach PS. Animal poisons in the tropics. In: Guerrant RL, Walker DH, Weller $\mathrm{PF}$, editors. Tropical infectious diseases: Principles, pathogens \& practice. Edinburgh: Churchill Livingstone; 2005. p.83-8.

3. Lévano J, Fernández R. Diagnóstico y tratamiento de los accidentes por animales ponzoñosos. Lima: Instituto Nacional de Salud; 2004.

4. Ministerio de Salud. Norma técnica sobre prevención $\mathrm{y}$ tratamiento de accidentes por animales ponzoñosos. Lima, Perú: Ministerio de Salud; 2019.
5. Kasturiratne A, Wickremasinghe AR, de Silva N, et al. The global burden of snakebite: A literature analysis and modelling based on regional estimates of envenoming and deaths. PLoS Med. 2008; 5:1591604.

6. Centro Nacional de Epidemiologia, Prevención y Control de Enfermedades. Enfermedades sujetas a vigilancia epidemiológica, Perú 2017. Boletin del Centro Nacional de Epidemiologia, Prevencion y Control de Enfermedades. 2017; 38:1268.

7. Villanueva M, Maguiña C, Cabada M, Demarini J, Álvarez H, Gotuzzo E. Ofidismo en la provincia de Chanchamayo, Junín: Revisión de 170 casos consecutivos en el Hospital de Apoyo de La Merced. Rev Med Hered. 2004; 15(2):82-7.

8. Zavaleta A. Mordedura de serpiente (ofidismo): un problema de salud en el Perú. Rev Med Hered. 2004; 15(2):61-3.

9. Demarini J. Ofidismo: aspectos etiológicos, clínicos y terapéuticos. Lima: Universidad Peruana Cayetano Heredia; 1992.

10. Gutiérrez JM, Calvete JJ, Habib AG, Harrison RA, Williams DJ, Warrell DA. Snakebite envenoming. Nat Rev Dis Primers. 2017; 3:17063. doi: 10.1038/ nrdp.2017.63

11. Yarlequé A, Vivas D, Rodríguez E, Sandoval GA, Pessah S, Bonilla C. Acción del antiveneno botrópico polivalente sobre las actividades proteolíticas presentes en los venenos de serpientes peruanas. Rev Peru Med Exp Salud Publica. 2008; 25(2):169-73.

12. Martín-Sierra C, Nogué-Xarau S, Pinillos MÁ, Rey JM. Envenenamiento por mordedura de serpiente en España. Emergencias. 2018; 30(2):126-132.

13. Alburqueeue P, Jacinto C, Silva Junior GB, Lima JB, Veras MS, Daher E. Acute kidney injury caused by Crotalus and Bothrops Snake Venom: Review of Epidemiology, clinical manifestations and treatment. Rev Inst Med Trop Sao Paulo. 2013; 55(5):295-301.

14. Centro Nacional de Epidemiologia, Prevención y Control de Enfermedades. Sala situacional para el Análisis de Situación de Salud - SE 27-2019. Lima: Centro Nacional de Epidemiologia, Prevención y Control de Enfermedades; 2019. (Citado el 29 de enero del 2020) Disponible en: http://www.dge.gob. pe/portal/docs/vigilancia/sala/2019/salaSE27.zip

Recibido: $19 / 08 / 2019$

Aceptado: 30/12/2019 